\title{
Pattern Motion Selectivity of Spiking Outputs and Local Field Potentials in Macaque Visual Cortex
}

\author{
Farhan A. Khawaja, James M. G. Tsui, and Christopher C. Pack \\ Montreal Neurological Institute, McGill University, Montreal, Quebec H3A 2B4, Canada
}

The dorsal pathway of the primate visual cortex is involved in the processing of motion signals that are useful for perception and behavior. Along this pathway, motion information is first measured by the primary visual cortex (V1), which sends specialized projections to extrastriate regions such as the middle temporal area (MT). Previous work with plaid stimuli has shown that most V1 neurons respond to the individual components of moving stimuli, whereas some MT neurons are capable of estimating the global motion of the pattern. In this work, we show that the majority of neurons in the medial superior temporal area (MST), which receives input from MT, have this pattern-selective property. Interestingly, the local field potentials (LFPs) measured simultaneously with the spikes often exhibit properties similar to that of the presumptive feedforward input to each area: in the high-gamma frequency band, the LFPs in MST are as component selective as the spiking outputs of MT, and MT LFPs have plaid responses that are similar to the spiking outputs of V1. In the lower LFP frequency bands (beta and low gamma), component selectivity is very common, and pattern selectivity is almost entirely absent in both MT and MST. Together, these results suggest a surprisingly strong link between the sensory tuning of cortical LFPs and afferent inputs, with important implications for the interpretation of imaging studies and for models of cortical function.

\section{Introduction}

In the primate brain, peripheral signals from each sensory modality are processed by a distributed network of cortical regions. Anatomically, these regions are generally organized in a hierarchical manner, such that the spiking outputs of one area provide the inputs to the next. Within each cortical area, the spiking outputs of single neurons can be studied with high spatial and temporal resolution through extracellular recording, and the resulting data have provided important insights into the relationship between single-neuron outputs and behavior and perception (Parker and Newsome, 1998).

A classic example of the hierarchical processing of sensory information is the computation of motion direction selectivity in the primate visual cortex. Direction selectivity first emerges in the primary visual cortex (V1), and it appears to be computed from thalamic inputs that are not themselves direction selective (Hubel and Wiesel, 1968). Similarly, a representation of motion direction for complex stimulus patterns is found in the middle temporal area (MT), but not in the V1 neurons that project to MT (Movshon and Newsome, 1996). These examples suggest that an important function of hierarchical sensory processing is to build selectivity to progressively more complex and behaviorally relevant stimulus features.

Received June 15, 2009; revised Sept. 2, 2009; accepted Sept. 14, 2009.

This work was supported by Canadian Institutes for Health Research Grant MOP-79352 (C.C.P.). F.A.K. was supported by a fellowship from Fonds de la Recherche en Santé du Québec (No. Dossier 13159). J.M.G.T. was supported by National Science and Engineering Research Council Fellowship PGS D3-362469-2008. We are grateful to Julie Coursol and Cathy Hunt for technical assistance, to Dr. Theo Zanos for assistance with some of the data analysis, and to Drs. Daniel Guitton and Gilles Plourde for helpful comments on this manuscript.

Correspondence should be addressed to Christopher C. Pack, 3801 University Street, Room 896, Montreal, QCH3A 2B4, Canada. E-mail: christopher.pack@mcgill.ca.

DOI:10.1523/JNEUROSCI.2844-09.2009

Copyright $\odot 2009$ Society for Neuroscience $\quad$ 0270-6474/09/2913702-08\$15.00/0
The above-mentioned results come from electrophysiological studies of single-neuron spiking outputs. However, a second type of signal that can be measured extracellularly is the local field potential (LFP), which corresponds to the total synaptic activity near the tip of the recording electrode (Mitzdorf, 1985), as well as voltage-activated membrane potential oscillations and spike afterpotentials (Buzsáki, 2002). The content of the LFP signal reflects in part the input to a given brain region, whereas the spiking activity of a neuron or group of neurons contributes to the output that is relayed to other parts of the brain. Both types of signals are also influenced by local processing within the circuit from which the recording is obtained, and consequently it is difficult to determine the origin of any particular feature of the recorded response.

In this work, we examined the stimulus selectivity of LFPs and spiking outputs by recording from multiple stages along the dorsal visual pathway of the alert macaque monkey, using stimuli that evoke markedly different patterns of spiking responses in each cortical area. Surprisingly, our results show that the tuning of LFP signals measured at certain frequencies is far more consistent with the presumptive inputs of a given extrastriate cortical region than with its outputs. We suggest that this result may be helpful for computational modeling of the input-output transformations in sensory processing hierarchies and for the interpretation of imaging studies wherein the signals measured correlate strongly with the LFPs (Logothetis et al., 2001).

\section{Materials and Methods}

Electrophysiological recordings. Two rhesus macaque monkeys took part in the experiments. Both underwent a sterile surgical procedure to implant a headpost and recording cylinder, and after recovery monkeys were seated comfortably in a primate chair (Crist Instruments) and trained to fixate a small red spot on a computer monitor in return for a 
liquid reward. Eye position was monitored at $200 \mathrm{~Hz}$ with an infrared camera (SR Research) and required to be within $2^{\circ}$ of the fixation point in order for the reward to be dispensed. All aspects of the experiments were approved by the Animal Care Committee of the Montreal Neurological Institute and were conducted in compliance with regulations established by the Canadian Council on Animal Care.

We recorded from well isolated single neurons in areas V1, MT, and medial superior temporal (MST). Single waveforms were sorted on-line and then resorted off-line, using spike-sorting software (Plexon). Area MT was identified based on anatomical magnetic resonance imaging (MRI) scans, the prevalence of direction-selective neurons, and on the correlation between receptive field size and eccentricity. Area MST was always found to be several millimeters past MT during a posterior approach to the superior temporal sulcus. It also contained larger receptive fields that extended into the ipsilateral visual field, and a large number of neurons that responded to expanding and/or rotating stimuli in addition to the translating stimuli used in the tests of pattern selectivity. These optic flow responses suggest that most of our recordings were from the dorsal, rather than the ventral, portion of MST, but this has not been verified histologically.

LFPs and single units (SUs) were recorded simultaneously on the same electrodes. LFPs were filtered using an analog two-pole low-cut $(0.7 \mathrm{~Hz})$ and a four-pole high-cut $(170 \mathrm{~Hz})$ filter (Plexon) and were then digitized and sampled at $1 \mathrm{kHz}$. The $60 \mathrm{~Hz}$ line noise was removed on-line using a software-switchable analog two-pole low-cut filter (Plexon). However, the power spectra of a number of LFP traces still showed a peak at $60 \mathrm{~Hz}$ and its first harmonic. Thus, we removed this noise off-line by applying a Gaussian notch filter (width, $5 \mathrm{~Hz}$ ) whose peak corresponded to the peak of the line noise. Single-unit waveforms were sampled at $40 \mathrm{kHz}$.

Procedure and visual stimuli. On each trial, the animal acquired fixation, after which the stimulus appeared and remained stationary for 200 $\mathrm{ms}$. The stimulus then moved at a constant direction and speed for 500 ms. Stimuli were displayed at $85 \mathrm{~Hz}$ at a resolution of $1920 \times 1200$ pixels, and the viewing area subtended $70 \times 42^{\circ}$ of visual angle at a distance of 42 $\mathrm{cm}$. Sinusoidal gratings were displayed on a gray background (luminance of $70.3 \mathrm{~cd} / \mathrm{m}^{2}$ ), and plaids were constructed by superimposing two gratings of $50 \%$ contrast that differed in motion direction by $120^{\circ}$. For all stimuli, spatial frequency, temporal frequency, and stimulus size were optimized for each cell. Motion direction was sampled in $30^{\circ}$ steps for both grating and plaid stimuli. Each stimulus was repeated five times in blockwise random order.

Data analysis. Spikes and LFPs were averaged over a time period that spanned 120-500 ms after the onset of stimulus motion. This time period was chosen to exclude the early period when the component/pattern classification changes in many cells (Pack et al., 2001; Smith et al., 2005). Spike tuning curves were considered direction selective if their directionality index (DI $=$ preferred direction response/null direction response) was $>2$. LFP responses were quantified by measuring their mean power, which was computed by using the discrete Fourier transform function in Matlab (Mathworks).

To obtain the average power spectrum for the population of MST or MT LFP sites in Figure 3, we considered only those sites that were responsive (see below) from 15 to $140 \mathrm{~Hz}$ since the LFP responses were generally excited at these frequencies. To compute LFP power across time from 16 to $170 \mathrm{~Hz}$, we calculated the response to the preferred grating motion direction in a sliding time window of $70 \mathrm{~ms}$, which was moved using a step size of $5 \mathrm{~ms}$. The power for each time bin was normalized to the baseline period (70 ms of spontaneous activity before the stimulus presentation). The resulting "normalized power" (Ray et al., 2008) was then plotted in decibels (by taking the log of the normalized power and multiplying by 10). The LFP power across time for $4-15 \mathrm{~Hz}$ was computed by using a sliding time window of $250 \mathrm{~ms}$, which was moved using a step size of $5 \mathrm{~ms}$. For the visual display purposes of Figure 3, we resampled these time bins (from 94 time bins for the $250 \mathrm{~ms}$ time window to 130 bins for the $70 \mathrm{~ms}$ time window), and frequencies $\sim 60 \mathrm{~Hz}$ (which we filtered out) were interpolated. However, note that we excluded these frequencies $(56-64 \mathrm{~Hz})$ for all of our analyses. Frequencies were sampled at a resolution of $1 \mathrm{~Hz}$ and we smoothed along the frequency axis by using a moving average of $10 \mathrm{~Hz}$.
Tuning curves were quantified using a $z$-score relative to the mean and $\mathrm{SD}$ of the spontaneous power in each frequency band. This metric allows comparison of sensory responses across multiple frequencies for which the baseline response varies (Kayser et al., 2007). We divided the LFP frequency spectrum into the following bands, similar to those used in previous studies (Viswanathan and Freeman, 2007; Ray et al., 2008): $\theta$ $(4-8 \mathrm{~Hz}), \alpha(8-12 \mathrm{~Hz}), \beta(16-24 \mathrm{~Hz})$, low $\gamma\left(\gamma_{\mathrm{L}}\right)(25-55 \mathrm{~Hz})$, and high $\gamma$ $\left(\gamma_{\mathrm{H}}\right)(65-140 \mathrm{~Hz})$. Thus, the number of dots in each plot in Figure $5 a$ and in supplemental Figures 3, 5, and 7 (available at www.jneurosci.org as supplemental material) reflects the number of recordings that were responsive and tuned at the corresponding frequency band. Because the response properties differed across frequencies, the number of excluded sites (and hence the number of dots in these figures) changes from band to band. Nearly identical (but somewhat noisier) results were obtained if we performed all analyses on the raw power in the LFP signal.

SU and LFP responses to gratings and plaids were classified according to the $Z$-transformed partial correlation coefficients between the data and the component and pattern predictions (Tinsley et al., 2003; Smith et al., 2005) using the following equations (shown for the $z$-transformed pattern correlation):

$$
Z_{p}=0.5 *\left(\ln \frac{\left(1+\mathrm{PC}_{p}\right) /\left(1-\mathrm{PC}_{p}\right)}{\sqrt{1 /(n-3)}}\right),
$$

where $n$ corresponds to the number of motion directions (12 in our experiments) and $\mathrm{PC}_{p}$ is defined as follows:

$$
\mathrm{PC}_{p}=\frac{\left(\mathrm{RC}_{p}-\mathrm{RC}_{c} \mathrm{RC}_{c p}\right)}{\sqrt{\left(1-\mathrm{RC}_{c}^{2}\right)\left(1-\mathrm{RC}_{c p}^{2}\right)}},
$$

where $\mathrm{RC}_{p}$ and $\mathrm{RC}_{c}$ are the raw correlations between the data and the pattern prediction and component predictions, respectively, and $\mathrm{RC}_{c p}$ is the raw correlation between the two predictions. The $Z$-transformed component correlation $\left(Z_{c}\right)$ can be obtained by exchanging $\mathrm{PC}_{p}$ and $\mathrm{PC}_{c}$, and the partial correlation $\left(\mathrm{PC}_{c}\right)$ between the component prediction and the data can be obtained by replacing $\mathrm{RC}_{p}$ with $\mathrm{RC}_{c}$ in the above equations. Each of the $z$-transformed values was tested for significance according to the criterion of 1.28 , equivalent to $p=0.90$. The pattern index was defined for each cell as $Z_{p}-Z_{c}$.

Supplemental material. The supplemental data (available at www. jneurosci.org as supplemental material) contain additional examples of pattern selectivity in single-unit and LFP responses as well as some control analyses.

\section{Results}

Single-unit pattern selectivity along the dorsal visual pathway We recorded SU activity and LFPs from a total of 186 sites in areas V1 $(N=33)$, MT $(N=79)$, and MST $(N=74)$ of two alert macaque monkeys. From these sites, we isolated a total of 229 SUs (33 in V1, 103 in MT, and 93 in MST). Areas MT and MST are specialized for the processing of visual motion (Maunsell and Newsome, 1987), and both receive direct and indirect input from area V1 (also known as the primary visual cortex) (Maunsell and van Essen, 1983; Boussaoud et al., 1990). In each case, we determined the selectivity of the SU activity for visual motion by drifting a sinusoidal grating in 12 different directions spaced evenly around the circle. Cells that failed to respond to the stimulus or that lacked direction selectivity (defined in Materials and Methods) were excluded from the analysis.

Direction-selective neurons were further tested with a second stimulus, which was constructed by superimposing two gratings that moved in directions that were separated by $120^{\circ}$ (Fig. 1a). The resulting plaid stimulus is perceived as moving in a direction that is consistent with the motion of the entire pattern (Adelson and Movshon, 1982), indicating that human observers are capable of integrating the motion signals generated by the individual stimulus components. Previous work has 
shown that some neurons in MT exhibit similar behavior (Movshon et al., 1985; Rodman and Albright, 1989; Pack et al., 2001), since they have nearly identical tuning curves for the grating and plaid stimuli. These cells are called "pattern selective," because they respond to the motion of the stimulus pattern regardless of the motion of the components. In contrast, many neurons in MT and most neurons in V1 respond primarily to the individual grating components of the plaid stimulus. The resulting direction tuning curves are typically bilobed, and these cells are called "component selective” (Movshon et al., 1985). Figure $1 b$ shows hypothetical tuning curves for a component-selective (dashed line) and pattern-selective (solid line) neuron.

We categorized each neuron in our sample as pattern or component selective, using standard statistical criteria (Tinsley et al., 2003; Smith et al., 2005) (see Materials and Methods), and Figure $2 a-c$ shows the resulting distributions for areas V1, MT, and MST. Here, blue dots represent component-selective neurons, red dots represent pattern-selective neurons, and black dots represent neurons that could not be assigned statistically to either category. Consistent with previous findings obtained with the same stimuli (Movshon et al., 1985), our results show that cells in V1 were mostly component selective, whereas the MT population contained approximately equal proportions of all three categories of cells. A novel finding of the current study is that MST neurons are mostly pattern selective $(60 \%)$ and very rarely component selective (9\%).

The pattern selectivity of LFPs has not previously been studied, but there are, broadly speaking, two types of results that one might expect to observe. The first is that the distribution of LFP pattern selectivity in each area might be similar to that of the spiking activity in the same area. This would be consistent with a strong contribution of recurrent or lateral connectivity to the LFP signal. The other possibility is that the LFP responses might more closely resemble the tuning of the spiking outputs of other areas, which would be more consistent with a role for feedforward (or feedback) input in determining the tuning properties of the LFPs.

The distinction between these two predictions is clearest in MST, in which the majority of neurons is pattern selective, and component selectivity is quite rare. Because pattern-selective neurons have nearly the same tuning curves in response to gratings and plaids, the sum of many pattern-selective responses will never be component selective, as component selectivity depends on a large difference in the responses to the two stimuli (Fig. 1b). Thus, we reasoned that the existence of strong component selectivity in the LFPs of area MST would likely correspond to feedforward input from areas that are known to project to MST and to contain a predominance of component selectivity (Fig. 2a,b) (Movshon and Newsome, 1996; Gegenfurtner et al., 1997). We therefore examined the pattern selectivity of MST LFPs to determine whether such component selectivity could be found.

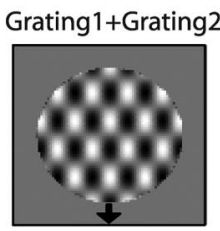

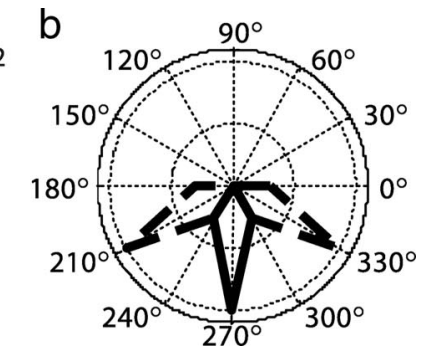

Figure 1. Component and pattern selectivity. $\boldsymbol{a}$, Examples of stimuli used in our experiments. Cells were stimulated with both single grating (left, center) and a plaid stimulus (right) formed by summing two gratings with orientations that differed by $120^{\circ}$. wo (dashed line) corresponding to the motions of the two component gratings.
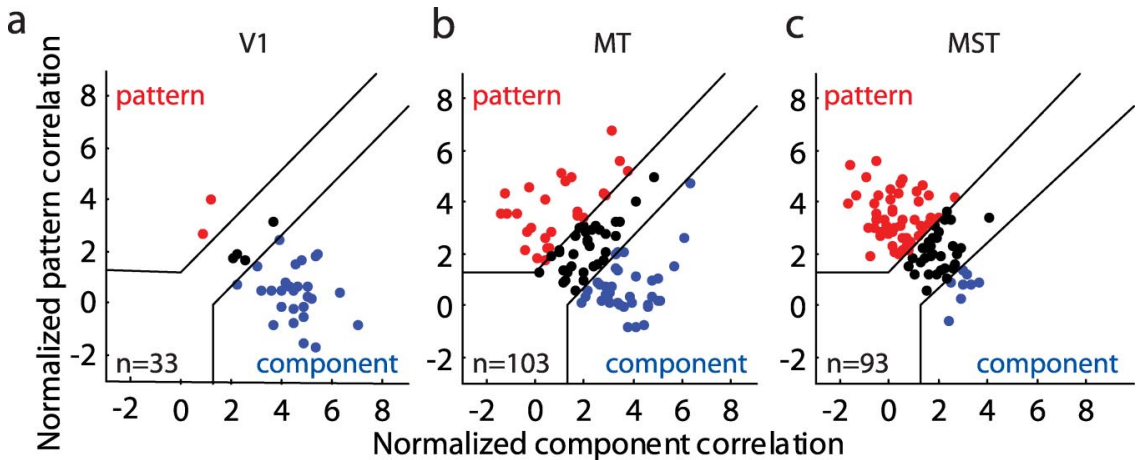

Normalized component correlation

Figure 2. Pattern and component selectivity in V1, MT, and MST. Scatter plots showing Z-transformed component correlations not be assigned to either category were labeled unclassified (black).

Pattern selectivity in local field potentials and spiking outputs We first grouped the LFP signals into different frequency bands chosen to be similar to those used in previous studies (Viswanathan and Freeman, 2007; Ray et al., 2008): $\theta(4-8 \mathrm{~Hz}), \alpha(8-12 \mathrm{~Hz}), \beta(16-24$ $\mathrm{Hz}), \gamma_{\mathrm{L}}(25-55 \mathrm{~Hz})$, and $\gamma_{\mathrm{H}}(65-140 \mathrm{~Hz})$. Within each band, we excluded LFPs that were not significantly modulated by visual stimulation (for exclusion criteria, see Materials and Methods). For the remaining sites, responses varied with frequency and time, as shown for grating and plaid stimuli in Figure 3 for areas MT and MST. Here, the color in each plot corresponds to the strength of the mean LFP activation, measured in decibels (see Materials and Methods). LFPs in both areas were modulated by the stimulus in a manner qualitatively similar to that observed previously in MT (Liu and Newsome, 2006; Viswanathan and Freeman, 2007). Specifically, the visual stimulus evokes an increase in power over a broad range of frequencies with a peak in the $\gamma_{\mathrm{H}}$ range (supplemental Fig. 1, available at www.jneurosci.org as supplemental material). Similar results have been reported in secondary somatosensory cortex (Ray et al., 2008), although other studies have found a peak in the $\gamma_{\mathrm{L}}$ band near $40 \mathrm{~Hz}$ (Pesaran et al., 2002; Henrie and Shapley, 2005; Viswanathan and Freeman, 2007). Because these studies involved different brain regions, anesthetic regimens, and behavioral tasks, it is difficult at present to determine which factors are responsible for the different peaks in the LFP frequency bands.

Consistent with a previous study in MT (Liu and Newsome, 2006), we found that most recording sites exhibited direction- 

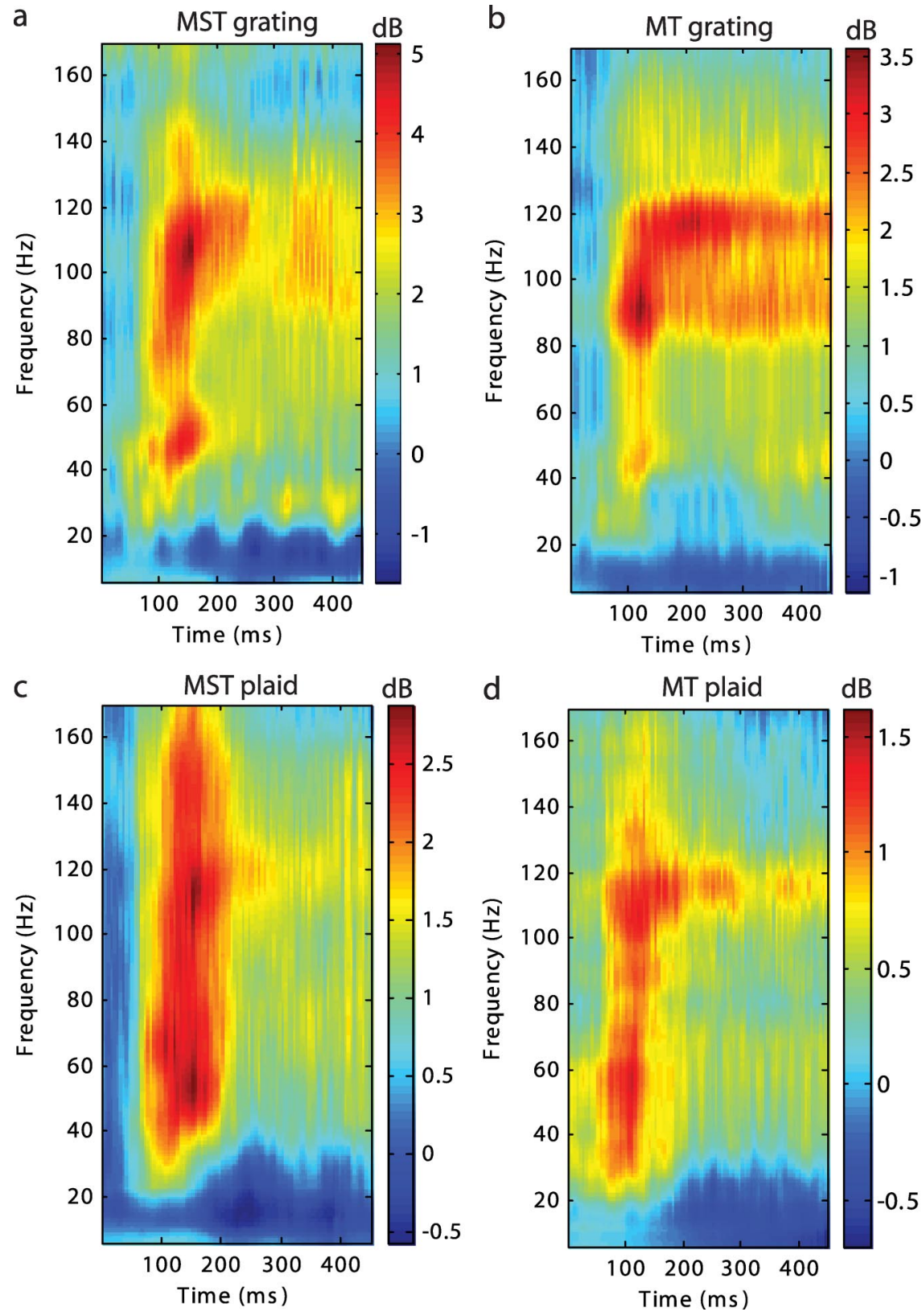

Figure 3. LFP spectrograms for MST and MT. Spectrograms showing the mean LFP power (across time and frequency) relative to baseline for the population of responsive sites in MST $(\boldsymbol{a})$ and MT $(\boldsymbol{b})$ in response to a sine wave grating stimulus. MST and MT spectrograms in response to a plaid stimulus are shown in c and $\boldsymbol{d}$, respectively. The color in each plot corresponds to the strength of the LFP activation measured in decibels.

tuned LFPs, and we analyzed these signals further to determine their component and pattern selectivity. Figure 4 shows the spiking responses (top) obtained from a single MST neuron, along with some of the LFP signals recorded from the same electrode (bottom). The left-hand column shows the responses to a grating stimulus, and the right-hand column shows the responses to the plaid. Although the SU activity and the LFP responses in the $\gamma_{\mathrm{H}}$ band are strongly pattern selective [pattern index (defined in Materials and Methods) $=1.79$ for SU and 4.46 for the $\gamma_{\mathrm{H}}$ band], the LFP response in the $\gamma_{\mathrm{L}}$ frequency band $(25-55 \mathrm{~Hz})$ is clearly component selective (pattern index $=-1.86$ ), since the tuning curve exhibits a separate peak for each of the two component gratings. Responses in the other LFP frequency bands $(\theta, \alpha$, and $\beta)$ for this site were mostly unresponsive or poorly tuned (data not shown).
As we show below, different recording sites had well tuned responses in different bands, and supplemental Table 1 (available at www. jneurosci.org as supplemental material) shows a summary of the percentages of tuned, untuned, and unresponsive sites for different bands of the LFP spectrum. Additional examples of recordings from individual sites in MT and MST are shown in supplemental Figure 2 (available at www. jneurosci.org as supplemental material).

\section{Pattern selectivity across LFP frequency bands}

The example in Figure 4 suggests that the tuning of the LFPs in certain frequency bands is similar to the spiking outputs of neurons in lower cortical areas. To examine this issue further, we computed the pattern selectivity for each MST recording for various frequency bands. For each recording site, we selected the frequency bands that were responsive to the stimulus and tuned to grating motion (see Materials and Methods), and computed direction tuning curves for both grating and plaid stimuli. Figure $5 a$, top row, shows the results for the different frequencies. Component selectivity (blue dots) dominates the low-frequency bands, with pattern selectivity (red dots) being found commonly only in the $\gamma_{\mathrm{H}}$ band. Of the sites that can be unambiguously classified as component or pattern selective, component selectivity ranges from a low of $53 \%$ in the $\gamma_{\mathrm{H}}$ band to a high of $100 \%$ in the $\beta$ band. The average value across all LFP bands was $75 \%$, compared with $13 \%$ for the SU responses. Pattern selectivity increased significantly from the $\gamma_{\mathrm{L}}$ band to the $\gamma_{\mathrm{H}}$ band $(t$ test, $p<0.001)$ and from the $\gamma_{\mathrm{H}}$ band to SUs ( $t$ test, $p<0.0001$ ). The proportions of component- and patternselective LFP responses did not change appreciably if we included LFP recording sites that did not meet our direction selectivity criterion (supplemental Fig. 3, available at www.jneurosci.org as supplemental material).

The strong component selectivity found in the LFPs would not be expected from any combination of the spiking outputs of the MST population, as the summed output of many pattern-selective neurons cannot be component selective. As mentioned above, the reason for this is that patternselective neurons by definition respond similarly to gratings and plaids, whereas component selectivity occurs only when there is a large difference between the responses to the two stimulus types. The component selectivity found in the MST LFPs is also not a consequence of noisy LFP signals, since additive noise would cause the responses to be categorized as unclassified, rather than component or pattern selective. Instead, we suggest that the high incidence of component selectivity found in MST LFPs is likely to be inherited from lower cortical areas. However, an alternative explanation for these results is that our single-unit sampling 
procedure was biased toward patternselective neurons in MST. Indeed, extracellular recordings typically oversample neurons with large cell bodies, and so if there were a positive correlation between pattern selectivity and cell size, our recordings might have missed componentselective neurons in MST. However, such neurons would still influence the LFPs, which are sensitive to all neuronal activity near the tip of the electrode.

Although we do not have a direct measure of the morphology of the single neurons from which we recorded, we can estimate the size of the cell body of each neuron based on the width of the corresponding action potential waveform (McCormick et al., 1985; Kawaguchi and Kubota, 1997; Nowak et al., 2003; Mitchell et al., 2007). We therefore plotted this latter value against the pattern index [a scalar measure of pattern selectivity (Smith et al., 2005) defined in Materials and Methods] for the SU responses in MST, and the results are shown in supplemental Figure 4 (available at www.jneurosci. org as supplemental material). There is no correlation between these measures $(p=$ $0.67)$. Thus, to the extent that waveform shape is a measure of cell size, any small neurons missed by our SU recordings would be expected to have the same distribution of pattern selectivity as the neurons from which we recorded.

Another possible explanation for our results is that the small fraction (9\%) of the component-selective SUs in MST exerted a disproportionate influence on the LFPs. This could occur if the component-selective neurons fired at a higher rate than their neighbors or if they exhibited much greater synchrony. The first possibility can be discarded, since the firing rate of component-selective MST neurons was on average significantly less than that of pattern-selective neurons ( $t$ test, $p<0.02$ ). The second possibility would be unexpected based on a previous study (Thiele and Stoner, 2003) that found little synchrony between pairs of component-selective cells that were stimulated with a perceptually coherent plaid. However, that study did not determine the prevalence of synchrony across layers, and this may be important for reasons that are mentioned in Discussion.

A final point to consider is the temporal dynamics of the responses to plaid stimuli. Previous work has shown that component selectivity dominates the early, transient phase of the response, even in cells that are classified as pattern selective when responses are averaged over longer time periods (Pack et al., 2001; Smith et al., 2005). If such transient responses exerted a strong influence on the LFPs (perhaps by being more synchronous), component selectivity might be overestimated in these responses. To examine this possibility, we excluded the transient phase of the responses and recalculated the degree of pattern selectivity using only the last $250 \mathrm{~ms}$ of the response in all of our MST recordings. The resulting distribution of pattern and component selectivity (supplemental Fig. 5, available at www.jneurosci.org as supple- mental material) changed very little, and the main result-strong component selectivity in the $\beta$ and $\gamma_{\mathrm{L}}$ LFP bands-remained unchanged.

We conclude that the low-frequency LFP responses in MST are primarily component selective, and that the most likely source of this selectivity is feedforward input from lower cortical (and perhaps subcortical) areas. Consistent with this idea, the LFPs in MT are almost entirely component selective (Fig. 5a, bottom row), as is the feedforward input to this area (Movshon and Newsome, 1996). Figure $5 b$ summarizes the pattern selectivity of the LFPs and the SU responses in both MT and MST.

The higher pattern selectivity found in the $\gamma_{\mathrm{H}}$ band of the LFPs in MST could reflect a mixture of both recurrent and feedforward contributions. It does not appear to be a consequence of contamination of the LFPs by the spikes, since we found no correlation between the pattern indices in the SUs and the high-gamma LFPs on a site-by-site basis $(p=0.74)$ (supplemental Fig. 6, available at www.jneurosci. org as supplemental material), and little spike-LFP coherence at the higher LFP frequencies (data not shown). At present, we do not have any way to identify the source of these gamma $\mathrm{H}_{\mathrm{H}} \mathrm{LF}$ responses, but we note that the responses at these frequencies in MT are almost entirely component selective (Fig. $5 a$, bottom right panel), and hence more consistent with feedforward than with recurrent input. The overall trend in the data does not change if we restrict our analysis to recordings for which the stimulus size was the same $\left(12^{\circ}\right)$ in 

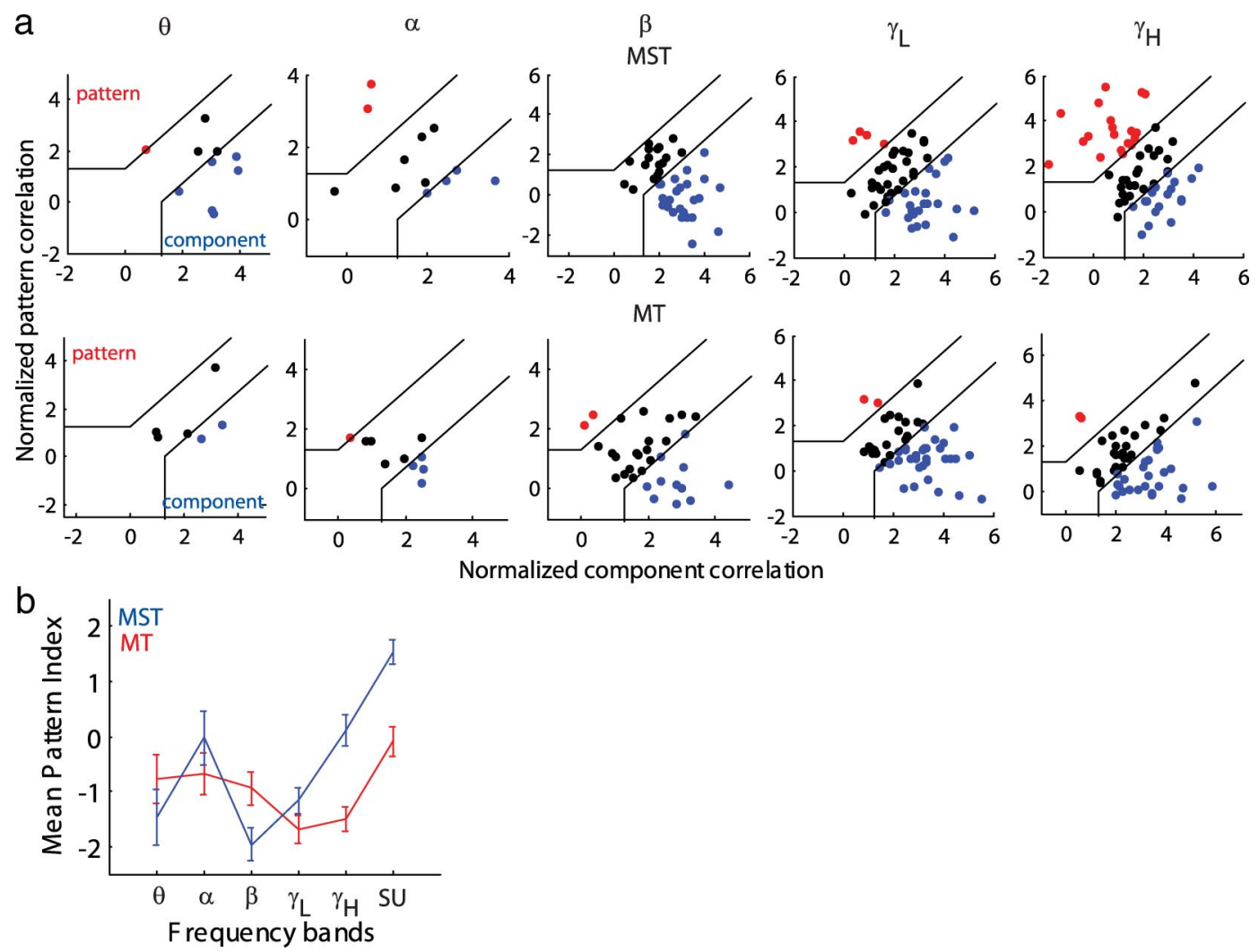

Figure 5. Component and pattern selectivity for tuned local field potentials recorded from areas MST and MT. $\boldsymbol{a}$, Scatter plots showing Z-transformed component correlations versus $Z$-transformed pattern correlations for tuned LFP signals in the $\theta, \alpha, \beta, \gamma_{L^{\prime}}$ and $\gamma_{\mathrm{H}}$ frequency bands for area MST (top) and MT (bottom). Conventions are as in Figure 2. $\boldsymbol{b}$, Plot showing the mean pattern index for responsive and tuned MST (blue) and MT (red) sites measured across the $\theta, \alpha, \beta, \gamma_{\mathrm{L}}$, and $\gamma_{\mathrm{H}}$ frequency bands and for single units. Error bars represent 1 SEM.

MT and MST (supplemental Fig. 7, available at www.jneurosci.org as supplemental material).

\section{Discussion}

We found that single-unit responses in area MST are mostly pattern selective, with component selectivity being quite rare. In contrast, local field potential signals in MST are often component selective, as are many of the neurons in areas that project to MST (Movshon and Newsome, 1996). A similar correspondence between the selectivity of the LFP signals and the spiking outputs of neurons in lower cortical areas was found in MT. These results are consistent with the idea that the $\beta, \gamma_{\mathrm{L}}$, and $\gamma_{\mathrm{H}}$ frequency bands of the LFP signal are heavily influenced by feedforward visual signals, although this conclusion is subject to a few caveats that we present here.

\section{Other potential sources of component selectivity in MST}

We have interpreted the strong component selectivity in the $\beta, \gamma_{L}$, and $\gamma_{\mathrm{H}}$ frequency bands as indicative of feedforward sensory input. As mentioned in Results, the component selectivity found in the MST LFPs is unlikely to come from within MST because component-selective single-neuron responses in that area were low in amplitude and quite rare (Fig. 2). Although it is possible that our extracellular recordings failed to detect component-selective neurons with small cell bodies, we believe that such a bias is unlikely to contribute substantially to our results for two reasons. First, although cell size correlates with the widths of action potential waveforms (McCormick et al., 1985; Kawaguchi and Kubota, 1997; Nowak et al., 2003; Mitchell et al., 2007), there was no correlation between waveform width and pattern selectivity (supplemental Fig. 4, available at www.jneurosci.org as supplemental material). Thus, any neurons that were invisible to our recordings were likely to be as pattern selective as the neurons from which we recorded. Second, the sheer magnitude of the component-selective contribution to the LFPs is difficult to reconcile with an account based on electrode bias. In the $\beta$ and $\gamma_{\mathrm{L}}$ bands, component selectivity in MST is $\sim 10$ times as common as pattern selectivity, whereas pattern-selective responses dominate the single-unit recordings.

Although cell size correlates poorly with pattern selectivity, it remains possible that there is a functional distinction between cells that exhibit component and pattern selectivity. Specifically, it is possible that interneurons are primarily component selective and that our recordings were mostly obtained from excitatory neurons. However, because interneurons likely contribute primarily to the high-gamma bands of the LFPs (Traub et al., 1996; Wang and Buzsáki, 1996; Galarreta and Hestrin, 1999; Tamás et al., 2000; Brunel and Wang, 2003; Whittington and Traub, 2003; Buzsáki and Draguhn, 2004; Tiesinga and Sejnowski, 2004; Hasenstaub et al., 2005; Henrie and Shapley, 2005; Gieselmann and Thiele, 2008), this hypothesis predicts a greater proportion of component selectivity in the higher frequency bands. In fact, we observe the opposite. Furthermore, such neurons are rare and poorly tuned (Nowak et al., 2008), whereas component selectivity is associated with tight direction tuning (Tinsley et al., 2003). Thus, we suggest that feedforward visual signals play an important role in shaping the tuning properties of LFPs. In MST, we find that at least $50 \%$ of all sites for which selectivity can be unambiguously classified exhibit component selectivity similar 
to that found in lower cortical areas, and this figure increases to nearly $100 \%$ in the lower $\left(\beta\right.$ and $\left.\gamma_{L}\right)$ bands.

\section{Implications for the interpretation of the LFP signal}

Our results are generally consistent with the idea that lowfrequency LFPs reflect synchrony in the synaptic inputs, whereas the higher frequencies are more sensitive to neuronal outputs (Niessing et al., 2005; Viswanathan and Freeman, 2007; Ray et al., 2008). They are also consistent with previous findings (Belitski et al., 2008) indicating that the low-frequency bands of the LFP signal convey stimulus-related information that differs from that found in the higher frequencies. Such low-frequency signals are thought to arise from distant brain regions (Belitski et al., 2008) that might include both cortical and subcortical areas. As mentioned above, the nature of the cortical signals is most consistent with visual areas that have little or no pattern selectivity. The potential subcortical contribution is more difficult to discern, because direction selectivity in subcortical areas has seldom been reported in the primate. In the cat, neurons in the lateral-posterior pulvinar (Merabet et al., 1998) are often pattern selective, which suggests that the homologous structure in the primate is unlikely to be the source of the component-selective LFPs we recorded in MT and MST.

Higher-frequency LFP signals are thought to originate within more local circuits (Henrie and Shapley, 2005; Belitski et al., 2008). Our results in MST are somewhat consistent with this idea, as we find the highest incidence of pattern selectivity in highgamma LFP frequencies. However, component selectivity is generally quite common even at these higher frequencies in both MT (Fig. $5 a$, bottom) and MST (Fig. $5 a$, top), which is consistent with a strong contribution of feedforward inputs to LFPs at these frequencies as well. Finally, we cannot completely exclude the possibility that LFP responses at the higher frequencies were corrupted by local spiking activity, in which case we will have actually overestimated the prevalence of pattern selectivity in MST LFPs.

The apparently dominant contribution of feedforward inputs to the tuning of the low-frequency LFP signal is surprising given that we made no effort to sample from the input layers of MT or MST in our recordings. Indeed, we have no accurate way of assigning our recordings to any particular layer. However, one speculative explanation for the strong influence of input signals on the LFPs is that multiple neurons in areas MT and MST receive input from the same neurons in areas like V1 and V2. In this scenario, divergent outputs from one area leads to increased synchrony in the input layers of the areas to which they project (Alonso et al., 1996), which in turn would cause an overrepresentation of feedforward visual signals in the LFPs.

\section{Implications for visual processing}

Although most V1 neurons are component selective (Fig. 2a), those that have strong surround suppression are capable of computing the global motion direction for stimuli that have twodimensional spatial features (Pack et al., 2003). Such neurons could in principle encode pattern motion by responding to the intersections of the two gratings in the plaid stimuli (Tinsley et al., 2003), and indeed the two pattern-selective V1 neurons from which we recorded were the most strongly surround-suppressed in our sample. This idea could be confirmed by testing V1 neurons with stimuli containing various types of two-dimensional features.

The computation of pattern motion from componentselective inputs has been studied extensively using psychophysics
(Movshon et al., 1985), single-unit electrophysiology (Movshon and Newsome, 1996), functional MRI (fMRI) (Huk and Heeger, 2002), and computational modeling (Rust et al., 2006). Our results on the pattern selectivity of LFPs and SU activity provide a means of linking some of these levels of investigation. In particular, theoretical studies have generally concluded that patternselective MT neurons represent a more sophisticated stage of motion processing than component-selective neurons, and thus one might expect them to be more prominently represented in the output of area MT. However, our results suggest that pattern selectivity is not especially common in the input to MST. This probably reflects the fact that MST receives input from many areas other than MT (Boussaoud et al., 1990), which means that the visual system must compute pattern motion at multiple locations in parallel, rather than simply relaying it from lower to higher areas. Alternatively, component-selective input may be useful for segmenting the motions of multiple objects (Stoner and Albright, 1992). Because our data represent the first exploration of pattern selectivity beyond $\mathrm{MT}$, it will be interesting to determine whether the increased pattern selectivity we found in MST reflects a stronger correlation with conscious perception (Williams et al., 2003) or simply a greater capacity for signal integration.

Previous work has shown that the blood oxygen leveldependent (BOLD) signal measured in fMRI experiments correlates well with LFPs (Logothetis et al., 2001). Thus, our results suggest that the pattern selectivity reported in human hMT+ (Huk and Heeger, 2002) originates in MST, since we found that pattern selectivity was extremely rare in MT LFPs (Fig. 5). Interestingly, the frequency band $\left(\gamma_{\mathrm{H}}\right)$ in which pattern selectivity is commonly found in MST is also responsive to the plaid components, suggesting that at least some of this activity originates in the input to this area. This result is an example of a more general point: when drawing comparisons between single-unit and fMRI data, it is important to consider the possibility that the latter reflect computations found in the spiking activity of brain regions that are quite distant from the source of the BOLD signal.

In presenting our data, we have excluded recordings that were not direction tuned, and so our results do not address the contribution of untuned modulatory inputs to the LFP signal. Such signals are likely to be critical for regulating contrast gain control (Shapley and Victor, 1981), controlling voluntary attention (Reynolds et al., 2000; Martínez-Trujillo and Treue, 2002), and normalizing neuronal outputs (Grossberg, 1973; Heeger, 1992), all of which may be important for the measurement of pattern motion in particular (Rust et al., 2006) and for cortical computation in general. However, such signals are common at every stage of visual processing, and so they are not useful for dissociating inputs from outputs, as we have attempted to do here.

\section{References}

Adelson EH, Movshon JA (1982) Phenomenal coherence of moving visual patterns. Nature 300:523-525.

Alonso JM, Usrey WM, Reid RC (1996) Precisely correlated firing in cells of the lateral geniculate nucleus. Nature 383:815-819.

Belitski A, Gretton A, Magri C, Murayama Y, Montemurro MA, Logothetis NK, Panzeri S (2008) Low-frequency local field potentials and spikes in primary visual cortex convey independent visual information. J Neurosci 28:5696-5709.

Boussaoud D, Ungerleider LG, Desimone R (1990) Pathways for motion analysis: cortical connections of the medial superior temporal and fundus of the superior temporal visual areas in the macaque. J Comp Neurol 296:462-495.

Brunel N, Wang XJ (2003) What determines the frequency of fast network 
oscillations with irregular neural discharges? I. Synaptic dynamics and excitation-inhibition balance. J Neurophysiol 90:415-430.

Buzsáki G (2002) Theta oscillations in the hippocampus. Neuron 33:325-340.

Buzsáki G, Draguhn A (2004) Neuronal oscillations in cortical networks. Science 304:1926-1929.

Galarreta M, Hestrin S (1999) A network of fast-spiking cells in the neocortex connected by electrical synapses. Nature 402:72-75.

Gegenfurtner KR, Kiper DC, Levitt JB (1997) Functional properties of neurons in macaque area V3. J Neurophysiol 77:1906-1923.

Gieselmann MA, Thiele A (2008) Comparison of spatial integration and surround suppression characteristics in spiking activity and the local field potential in macaque V1. Eur J Neurosci 28:447-459.

Grossberg S (1973) Contour enhancement, short-term memory, and constancies in reverberating neural networks. Stud Appl Math 52:213-257.

Hasenstaub A, Shu Y, Haider B, Kraushaar U, Duque A, McCormick DA (2005) Inhibitory postsynaptic potentials carry synchronized frequency information in active cortical networks. Neuron 47:423-435.

Heeger DJ (1992) Normalization of cell responses in cat striate cortex. Vis Neurosci 9:181-197.

Henrie JA, Shapley R (2005) LFP power spectra in V1 cortex: the graded effect of stimulus contrast. J Neurophysiol 94:479-490.

Hubel DH, Wiesel TN (1968) Receptive fields and functional architecture of monkey striate cortex. J Physiol 195:215-243.

Huk AC, Heeger DJ (2002) Pattern-motion responses in human visual cortex. Nat Neurosci 5:72-75.

Kawaguchi Y, Kubota Y (1997) GABAergic cell subtypes and their synaptic connections in rat frontal cortex. Cereb Cortex 7:476-486.

Kayser C, Petkov CI, Logothetis NK (2007) Tuning to sound frequency in auditory field potentials. J Neurophysiol 98:1806-1809.

Liu J, Newsome WT (2006) Local field potential in cortical area MT: stimulus tuning and behavioral correlations. J Neurosci 26:7779-7790.

Logothetis NK, Pauls J, Augath M, Trinath T, Oeltermann A (2001) Neurophysiological investigation of the basis of the fMRI signal. Nature 412: $150-157$.

Martínez-Trujillo J, Treue S (2002) Attentional modulation strength in cortical area MT depends on stimulus contrast. Neuron 35:365-370.

Maunsell JH, Newsome WT (1987) Visual processing in monkey extrastriate cortex. Annu Rev Neurosci 10:363-401.

Maunsell JH, van Essen DC (1983) The connections of the middle temporal visual area (MT) and their relationship to a cortical hierarchy in the macaque monkey. J Neurosci 3:2563-2586.

McCormick DA, Connors BW, Lighthall JW, Prince DA (1985) Comparative electrophysiology of pyramidal and sparsely spiny stellate neurons of the neocortex. J Neurophysiol 54:782-806.

Merabet L, Desautels A, Minville K, Casanova C (1998) Motion integration in a thalamic visual nucleus. Nature 396:265-268.

Mitchell JF, Sundberg KA, Reynolds JH (2007) Differential attentiondependent response modulation across cell classes in macaque visual area V4. Neuron 55:131-141.

Mitzdorf U (1985) Current source-density method and application in cat cerebral cortex: investigation of evoked potentials and EEG phenomena. Physiol Rev 65:37-100.

Movshon JA, Newsome WT (1996) Visual response properties of striate cortical neurons projecting to area MT in macaque monkeys. J Neurosci 16:7733-7741.

Movshon JA, Adelson EH, Gizzi MS, Newsome WT (1985) The analysis of visual moving patterns. In: Pattern recognition mechanisms (Chagas C, Gatass R, Gross C, eds), pp 117-151. New York: Springer.

Niessing J, Ebisch B, Schmidt KE, Niessing M, Singer W, Galuske RA (2005) Hemodynamic signals correlate tightly with synchronized gamma oscillations. Science 309:948-951.
Nowak LG, Azouz R, Sanchez-Vives MV, Gray CM, McCormick DA (2003) Electrophysiological classes of cat primary visual cortical neurons in vivo as revealed by quantitative analyses. J Neurophysiol 89:1541-1566.

Nowak LG, Sanchez-Vives MV, McCormick DA (2008) Lack of orientation and direction selectivity in a subgroup of fast-spiking inhibitory interneurons: cellular and synaptic mechanisms and comparison with other electrophysiological cell types. Cereb Cortex 18:1058-1078.

Pack CC, Berezovskii VK, Born RT (2001) Dynamic properties of neurons in cortical area MT in alert and anaesthetized macaque monkeys. Nature 414:905-908.

Pack CC, Livingstone MS, Duffy KR, Born RT (2003) End-stopping and the aperture problem: two-dimensional motion signals in macaque V1. Neuron 39:671-680.

Parker AJ, Newsome WT (1998) Sense and the single neuron: probing the physiology of perception. Annu Rev Neurosci 21:227-277.

Pesaran B, Pezaris JS, Sahani M, Mitra PP, Andersen RA (2002) Temporal structure in neuronal activity during working memory in macaque parietal cortex. Nat Neurosci 5:805-811.

Ray S, Crone NE, Niebur E, Franaszczuk PJ, Hsiao SS (2008) Neural correlates of high-gamma oscillations $(60-200 \mathrm{~Hz})$ in macaque local field potentials and their potential implications in electrocorticography. J Neurosci 28:11526-11536.

Reynolds JH, Pasternak T, Desimone R (2000) Attention increases sensitivity of V4 neurons. Neuron 26:703-714.

Rodman HR, Albright TD (1989) Single-unit analysis of pattern-motion selective properties in the middle temporal visual area (MT). Exp Brain Res 75:53-64.

Rust NC, Mante V, Simoncelli EP, Movshon JA (2006) How MT cells analyze the motion of visual patterns. Nat Neurosci 9:1421-1431.

Shapley RM, Victor JD (1981) How the contrast gain control modifies the frequency responses of cat retinal ganglion cells. J Physiol 318:161-179.

Smith MA, Majaj NJ, Movshon JA (2005) Dynamics of motion signaling by neurons in macaque area MT. Nat Neurosci 8:220-228.

Stoner GR, Albright TD (1992) Neural correlates of perceptual motion coherence. Nature 358:412-414.

Tamás G, Buhl EH, Lörincz A, Somogyi P (2000) Proximally targeted GABAergic synapses and gap junctions synchronize cortical interneurons. Nat Neurosci 3:366-371.

Thiele A, Stoner G (2003) Neuronal synchrony does not correlate with motion coherence in cortical area MT. Nature 421:366-370.

Tiesinga PH, Sejnowski TJ (2004) Rapid temporal modulation of synchrony by competition in cortical interneuron networks. Neural Comput $16: 251-275$.

Tinsley CJ, Webb BS, Barraclough NE, Vincent CJ, Parker A, Derrington AM (2003) The nature of V1 neural responses to 2D moving patterns depends on receptive-field structure in the marmoset monkey. J Neurophysiol 90:930-937.

Traub RD, Whittington MA, Stanford IM, Jefferys JG (1996) A mechanism for generation of long-range synchronous fast oscillations in the cortex. Nature 383:621-624.

Viswanathan A, Freeman RD (2007) Neurometabolic coupling in cerebral cortex reflects synaptic more than spiking activity. Nat Neurosci 10:1308-1312.

Wang XJ, Buzsáki G (1996) Gamma oscillation by synaptic inhibition in a hippocampal interneuronal network model. J Neurosci 16:6402-6413.

Whittington MA, Traub RD (2003) Interneuron diversity series: inhibitory interneurons and network oscillations in vitro. Trends Neurosci 26: $676-682$.

Williams ZM, Elfar JC, Eskandar EN, Toth LJ, Assad JA (2003) Parietal activity and the perceived direction of ambiguous apparent motion. Nat Neurosci 6:616-623. 